\title{
Decay to the nonequilibrium steady state of the thermal diffusion in a tilted periodic potential
}

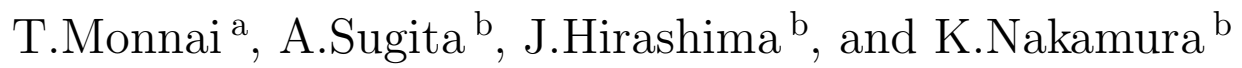 \\ ${ }^{a}$ Department of Applied Physics ,Waseda University, 3-4-1 Okubo, \\ Shinjuku-ku,Tokyo 169-8555,Japan \\ ${ }^{\mathrm{b}}$ Department of Applied Physics, Osaka City University, 3-3-138 Sugimoto, \\ Sumiyoshi-ku, Osaka 558-8585, Japan
}

\begin{abstract}
We investigate asymptotic decay phenomenon towards the nonequilibrium steady state of the thermal diffusion in the presence of a tilted periodic potential. The parameter dependence of the decay rate is revealed by investigating the Fokker-Planck (FP) equation in the low temperature case under the spatially periodic boundary condition (PBC). We apply the WKB method to the associated Schrödinger equation. While eigenvalues of the non-Hermitian FP operator are complex in general, in a small tilting case the imaginary parts of the eigenvalues are almost vanishing. Then the Schrödinger equation is solved with PBC. The decay rate is analyzed in the context of quantum tunneling through a triple-well effective periodic potential. In a large tilting case, the imaginary parts of the eigenvalues of FP operator are crucial. We apply the complex-valued WKB method to the Schrödinger equation with the absorbing boundary condition, finding that the decay rate saturates and depends only on the temperature, the potential periodicity and the viscous constant. The intermediate tilting case is also explored. The analytic results agree well with the numerical data for a wide range of tilting.
\end{abstract}

Key words: decay rate, thermal diffusion, tilted periodic potential, WKB analysis, Fokker-Planck equation

Email addresses: monnai@suou.waseda.jp (T.Monnai), sugita@a-phys.eng.osaka-cu.ac.jp (A.Sugita), nakamura@a-phys.eng. osaka-cu.ac.jp (K.Nakamura). 


\section{Introduction}

Thermal diffusion process in a periodic potential in the presence of external force appears in many situations. In solid-state physics, it appears in diffusion of ions or molecules on crystal surfaces[1], Josephson junctions[2], motion of fluxons in superconductors[3], rotation of molecules in solids[4], superionic conductors[5], charge density waves[6], to mention a few. As a ratchet system, it is also applied for particle selection[7], biophysical processes[8] and intracellular transport[9][10]. Most of the researches in this context are concerned with the steady state. For example, it is shown that a symmetric dichotomous noise causes non-zero steady state current in such a system[11]. The existence of an optimal diffusion coefficient vs. external force is also reported[12].

Such a diffusion process can be described by the over-damped Langevin equation with a tilted periodic potential,

$$
\begin{aligned}
\eta \dot{x}(t) & =-\frac{\partial}{\partial x} U^{(0)}(x)+\frac{2 \pi W}{L} x+\xi(t), \\
\langle\xi(t)\rangle & =0, \quad\left\langle\xi(t) \xi\left(t^{\prime}\right)\right\rangle=2 \eta \theta \delta\left(t-t^{\prime}\right) .
\end{aligned}
$$

Here, $\eta, \theta=k_{B} T$ and $W$ are the viscous coefficient, temperature and the external force, respectively. $\xi(t)$ is the Langevin force which satisfies the fluctuation dissipation theorem. The periodic potential $U^{(0)}(x+L)=U^{(0)}(x)$ with period $L$ is tilted by the external tilting force $F=\frac{2 \pi W}{L}$ and we call the potential $U(x) \equiv U^{(0)}(x)-\frac{2 \pi W}{L} x$ as a tilted potential.

In order to investigate the probability density $P(x, t)$ that the particle is found in a position $x$ at time $t$, it is useful to transform the Langevin equation into the corresponding Fokker-Planck(FP) equation,

$$
\frac{\partial}{\partial t} P(x, t)=\frac{1}{\eta} \frac{\partial}{\partial x}\left(\frac{\partial U(x)}{\partial x}+\theta \frac{\partial}{\partial x}\right) P(x, t) .
$$

Hereafter we solve FP equation (2) under the (spatially) periodic boundary condition (PBC), $P(x+L, t)=P(x, t)$, which is satisfied by the steady state at $t \rightarrow \infty$. The analytic expression for the steady state $P^{s t}(x) \equiv \lim _{t \rightarrow \infty} P(x, t)$ under PBC is well-known[9,13,14]:

$$
P^{s t}(x)=N \frac{\eta}{\theta} e^{-\frac{U(x)}{\theta}} \int_{x}^{x+L} d y e^{\frac{U(y)}{\theta}}
$$




$$
N=\frac{\theta}{\eta}\left(\int_{0}^{L} d x \int_{x}^{x+L} d y e^{\frac{U(y)-U(x)}{\theta}}\right)^{-1}
$$

where $N$ is the normalization constant chosen so that $\int_{0}^{L} d x P^{s t}(x)=1$. The steady state has a non-zero probability current $J^{s t}(x)$ given by

$$
J^{s t} \equiv \frac{1}{\eta}\left(U(x)-\theta \frac{\partial}{\partial x}\right) P^{s t}(x)=N\left(1-e^{-\frac{2 \pi W}{\theta}}\right) .
$$

The above steady state is a nonequilibrium state. In this paper, we study the low-temperature relaxation dynamics towards the steady state in the presence of a tilted periodic potential. In particular, asymptotic behaviors of the decay phenomenon is investigated by the eigenfunction expansion method. Our main interest lies in parameter dependence of the decay rate.

This paper is organized as follows. In section 2 we present the framework of our analysis, relevant numerical data for eigenvalues of the FP operator, and the main result. Section 3 treats the important theme on the boundary condition for eigenstates of the associated Schrödinger equation. In subsection 3.1 the small tilting case is investigated in the context of a tunneling problem. In subsection 3.2 the large tilting case is treated, where we shall apply the complex-valued WKB method to the Schrödinger equation with the absorbing boundary condition deduced from PBC for the original FP equation. Section 4 is devoted to a summary. Appendix is concerned with detailed calculations in section 3.2 .

\section{Eigenvalues of the Fokker-Planck operator}

The WKB treatment of FP equation has a long history since the monumental work by van Kampen[15]. Most of the studies, however, were limited to the system with a well-defined thermal equilibrium state[16]. This fact guaranteed a real-valued nature of eigenvalues for the non-Hermitian FP operator, and one can reduce FP equation to the eigenvalue problem of the associated Schrödinger equation with the same natural boundary condition as the FP equation has. On the other hand, relaxation dynamics towards the nonequilibrium steady state with the non-vanishing constant current demands a qualitatively different WKB approach. In fact FP operator in such a system has complex eigenvalues in general.

This situation is recognized in our system with a tilted sinusoidal potential[17],

$$
U(x)=U_{0} \cos \left(\frac{2 \pi x}{L}\right)-\frac{2 \pi W}{L} x
$$


where $U_{0}$ is the amplitude of the periodic part of the potential. Putting eigenvalues of the non-Hermitian FP operator as $-E$ and substituting $P(x, t)=$ $e^{-E t} P(x)$ into Eq.(2), we have the eigenvalue problem,

$$
-E P(x)=\frac{1}{\eta} \frac{\partial}{\partial x}\left(\frac{\partial U(x)}{\partial x}+\theta \frac{\partial}{\partial x}\right) P(x) .
$$

While the steady state in Eq.(3) is periodic, $P^{s t}(x+L)=P^{s t}(x)$, and is characterized by the zero Bloch wavenumber $(k=0)$, it is difficult to analyze the relaxation dynamics in general. Any initial distribution $P(x, 0)$ which include $k \neq 0$ Fourier components can relax towards $P^{s t}(x)$ : the $k \neq 0$ components will decay out on the way of time evolution. In the present work, however, we confine ourselves to the manifold of $k=0$, which makes the analysis of the decay phenomenon accessible. This implies that the time-dependent solution of FP equation in Eq.(2) relaxing towards the steady state should satisfy the spatial PBC, $P(x+L, t)=P(x, t)$ and be constructed from solutions with a natural boundary condition $Q(x, t)\left(\lim _{x \rightarrow \pm \infty} Q(x, t)=0\right)$ as

$$
P(x, t)=\sum_{n=-\infty}^{\infty} Q(x+n L, t)
$$

Since the FP operator in Eq.(2) is invariant against space translation by $L$, it has Bloch-type eigenstates $\left\{P_{n, k}(x)\right\}$ and (complex) eigenvalues $\left\{E_{n, k}\right\}$. Noting the normalization matrix $\int_{0}^{L} d x P_{k, n}^{*}(x) P_{k^{\prime}, n^{\prime}}(x)=\delta_{k, k^{\prime}} N_{n, n^{\prime}}^{(k)}$ due to the nonHermitian nature of FP operator, the solution $P(x, t)$ in Eq.(7) is now written explicitly as

$$
\begin{aligned}
& P(x, t)=\sum_{n} C_{n, k=0} P_{n, k=0}(x) e^{-E_{n, k=0} t}, \\
& C_{n, k=0}=\sum_{n^{\prime}}\left(N^{(0)}\right)_{n, n^{\prime}}^{-1} \int_{0}^{L} d x P_{n^{\prime}, k=0}^{*}(x) P(x, 0), \quad C_{n, k \neq 0}=0,
\end{aligned}
$$

which starts from an arbitrary $k=0$ distribution $P(x, 0)$ at $t=0$ and relaxes towards $P^{s t}(x)$.

In the manifold with $k=0$, the probability distribution can be expanded as $P(x)=\sum_{n} c_{n} e^{\frac{2 \pi i x}{L} n}$. Then the eigenvalue problem (6) is reduced to

$$
\begin{aligned}
& \left(\frac{2 \pi}{L}\right)^{2} \sum_{m} A_{n, m} c_{m}=\eta E c_{n} \\
& A_{n, m}=-\left(i n W+\theta n^{2}\right) \delta_{n, m}-\frac{n U_{0}}{2} \delta_{n-1, m}+\frac{n U_{0}}{2} \delta_{n+1, m}
\end{aligned}
$$




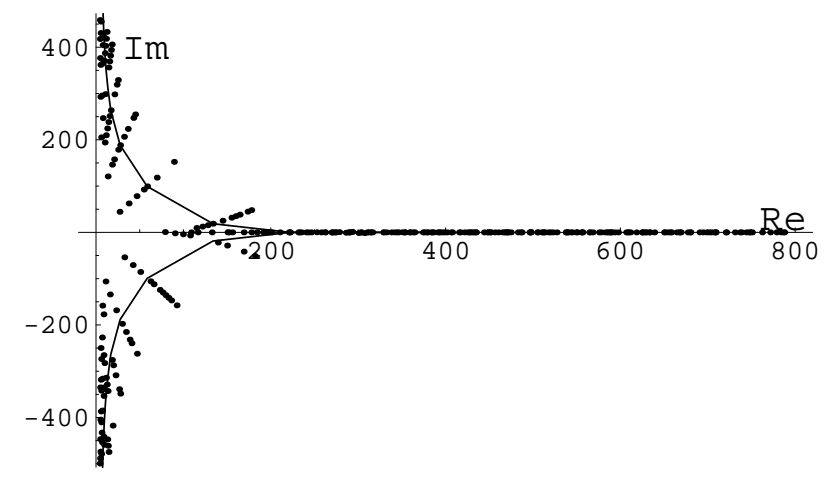

Fig. 1. Distribution of the complex eigenvalue with the second smallest real part for full parameter range $3 \leq U_{0} \leq 20,0 \leq W \leq 20$ (dots), and for the case of fixed potential barrier $U_{0}=10,0 \leq W \leq 20$ (solid line). A pair of solid lines rapidly merge to the real axis as $W$ decreases below $U_{0}$.

which can be solved numerically by truncating the infinite dimensional matrix $A$ to a large but finite dimendional matrix.

The zero-eigenvalue $(E=0)$ corresponds to the steady state. Under PBC, as is obvious from Eq. (8), the probability distribution approaches exponentially to the unique steady state, and the decay rate $\lambda$ is obtained as the real part of the second smallest eigenvalue. Among many complex eigenvalues of Eq.(10), therefore, we have focused on the one with the second-smallest real part and its variation against various parameter values $U_{0}, W$ (see Fig.1). From Fig.1 one can see that the eigenvalues with large positive real parts have nearly zero imaginary part. (See tails of solid lines in Fig. 1.) These eigenvalues correspond to the small tilting case. In fact, a pair of solid lines rapidly merge to the real axis as $W$ decreases below $U_{0}$, ensuring the real-valued nature of eigenvalues in the case of small tilting.

$U_{0}$ and $W$ dependence of the decay rate $\lambda$ is shown in Fig. 2, which we have confirmed by numerically solving the FP equation in Eq.(2). There is a smooth but steep decrease of the decay rate around the crossover line of $U_{0} \simeq W$, where the original potential minima disappear.

In the next section, the Schrödinger equation associated with FP equation will be solved with use of the WKB method. In the small tilting case in section 3.1, we impose PBC for the wavefunction of the Schrödinger equation and apply the Bloch theorem. The decay rate for $W<U_{0}$ is shown to be $\left(\frac{2 \pi}{L}\right)^{2} \sqrt{1-\left(\frac{W}{U_{0}}\right)^{2}} U_{0}$. In the large tilting case in subsection 3.2 , we have recourse to the absorbing boundary condition and apply the WKB method which admits complex energies. The decay rate for $W \gg U_{0}$ is found to be $(2 \pi / L)^{2} \theta$ (with no $W$ and $U_{0}$ dependence). The crossover region where $W$ is comparable to $U_{0}$ is numerically analyzed based on this approach. 


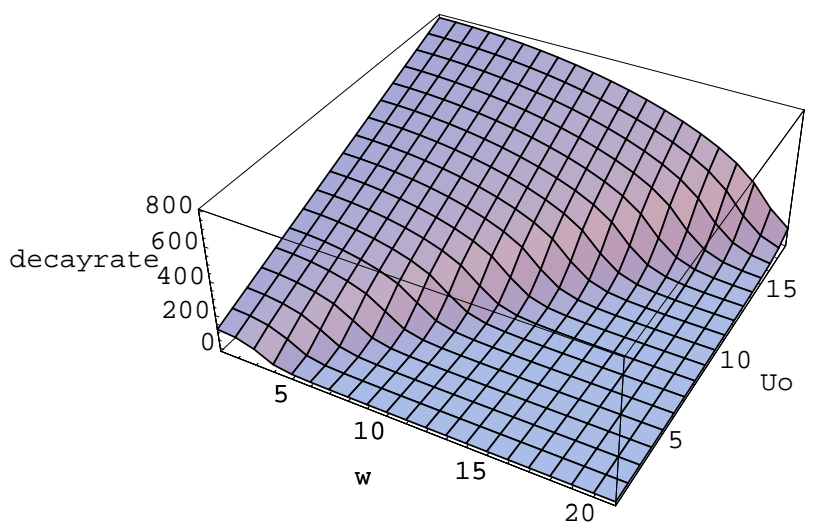

Fig. 2. $W$ and $U_{0}$ dependence of the decay rate $\lambda$.

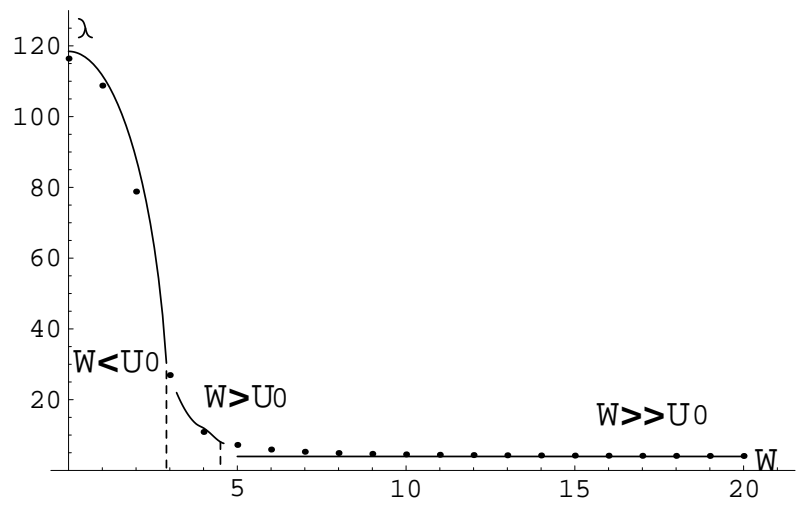

Fig. 3. $W$ dependence of the decay rate $\lambda$ for $U_{0}=3, \theta=0.1$. Dots show the decay rates obtained from numerical diagonalization of FP operator. Three solid lines are theoretical results (For small tilting case $W<U_{0}$ the decay rate $\lambda$ is obtained as the first excited level $\hbar \omega_{a}$ of the associated Schrödinger equation (left part). For crossover regime $W \sim U_{0}, \lambda$ is given as the numerical solution of (37)(middle part). For large tilting case $W \gg U_{0}$, the perturbative solution of (38) gives $\lambda$ (right part).

\section{WKB analysis of probability distribution}

The FP equation is transformed into the Schrödinger equation by the separation ansatz[15]

$$
P(x, t)=e^{-\frac{U(x)}{2 \theta}} \phi(x) e^{-E t}
$$

Then one has

$$
\theta \frac{d^{2}}{d x^{2}} \phi(x)+(E-V(x)) \phi(x)=0
$$


where we redefined eigenvalue as $E \eta \rightarrow E$, and defined the effective potential

$$
V(x) \equiv \theta\left\{\left(\frac{U^{\prime}(x)}{2 \theta}\right)^{2}-\frac{U^{\prime \prime}(x)}{2 \theta}\right\}
$$

The Schrödinger operator (SO) in Eq.(12) looks Hermitian. Noting in Eq.(11) $U(x+L)=U(x)-2 \pi W$ and $P_{n, k}(x+L)=e^{i k L} P_{n, k}(x)$, however, the wave function $\phi(x)$ of the Schrödinger equation should satisfy the absorbing boundary condition,

$$
\phi(x+L)=e^{i k L-\frac{2 \pi W}{2 \theta}} \phi(x)
$$

which eventually breaks the Hermitian nature of SO.

In the following we shall analyze the Schrödinger equation in Eq.(12) first in the small tilting case and then in the strong tilting case. In the small tilting case, the numerical evidence in the previous section has shown the real-valued nature of the second-lowest eigenvalue, indicating the recovery of Hermitian nature of SO. Therefore we reduce Eq. (14) to

$$
\phi(x+L)=e^{i k L} \phi(x)
$$

by assuming the absorbing term $\frac{2 \pi W}{2 \theta}$ being vanishing. The validity of this assumption will be verified by comparing between the analytic and numerical decay rates. Below we shall confine to the $k=0$ manifold.

\subsection{Small tilting case}

Substituting the tilted sinusoidal potential (5) into (13), one has the effective potential,

$$
V(x)=\frac{\pi^{2}}{L^{2} \theta}\left(U_{0} \sin \frac{2 \pi x}{L}+W\right)^{2}+\frac{1}{2}\left(\frac{2 \pi}{L}\right)^{2} U_{0} \cos \frac{2 \pi}{L} x .
$$

Since the first term is much larger than the second one in the low temperature regime $\theta \ll U_{0}$, the second one is omitted hereafter.

In solving Eq.(12) the low temperature condition allows us to use the WKB approximation, since $\theta$ corresponds to $\frac{\hbar^{2}}{2 m}$ in the standard Schrödinger equation. In the case that the external force is weak $\left(W<U_{0}\right)$, the effective potential 


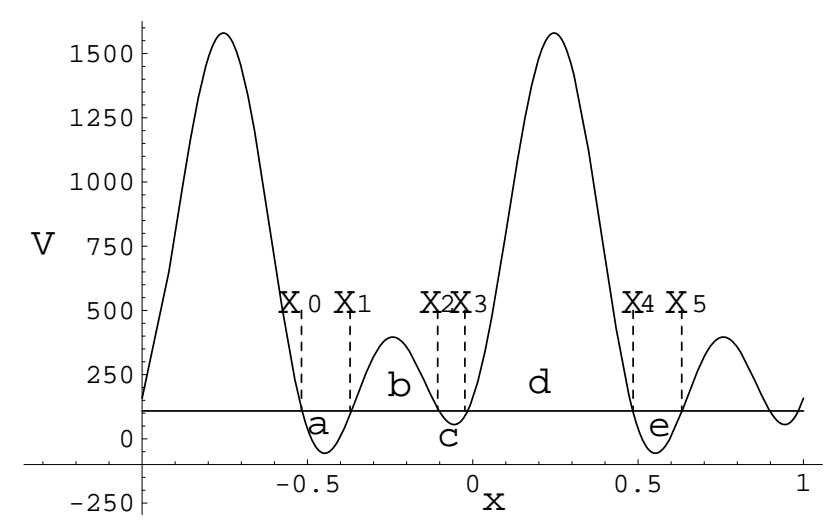

Fig. 4. Effective potential $V(x)$ and the real part of the eigenvalue of FP operator with second smallest real part $E$ with the corresponding classical turning points $x_{0}, \ldots, x_{5}$ for $W=1, U_{0}=3$.

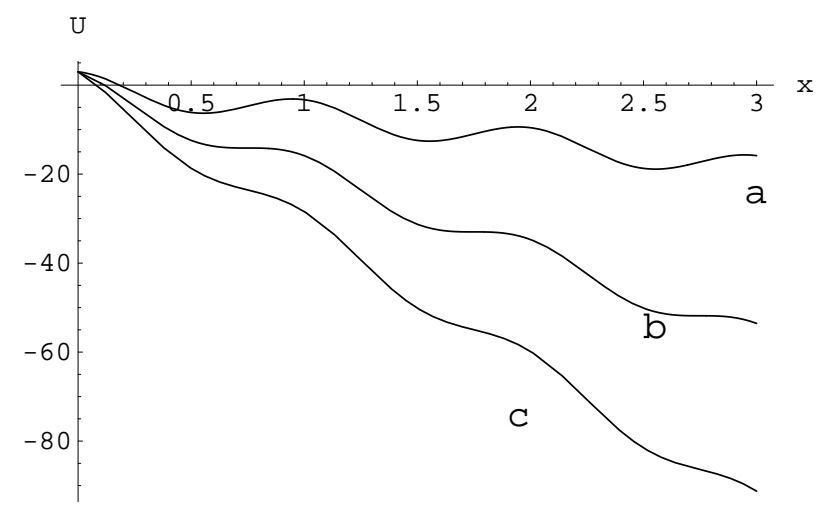

Fig. 5. Original tilted potential $U(x)$ in real space. Small tilting case (a), crossover region (b), and large tilting case (c).

$V(x)$ has three wells. We consider the energy levels for the eigenstates which experience all the three wells[18] (see Fig. 4).

In terms of the tilted potential $U(x)$, the weak external force guarantees the existence of potential minima in Fig. 5. Let us denote the wave functions in each region $a, \cdots, e$ bordered by classical turning points $x_{0}, \cdots, x_{5}$ as $\phi_{a}, \cdots, \phi_{e}$ and introduce the action integrals

$$
\begin{array}{ll}
S_{a}=\frac{1}{\hbar} \int_{x_{0}}^{x_{1}} p d x, & M_{b}=\frac{1}{\hbar} \int_{x_{1}}^{x_{2}} p d x \\
S_{c}=\frac{1}{\hbar} \int_{x_{2}}^{x_{3}} p d x, & M_{d}=\frac{1}{\hbar} \int_{x_{3}}^{x_{4}} p d x,
\end{array}
$$

where $\hbar^{2}=2 m \theta$ and $p=\sqrt{2 m|E-V(x)|}(p$ stands for the momentum in a potential-well region). $S$ and $M$ are defined in each well and barrier, respectively. Each neighboring wave functions are mutually related by the connection 
formula

$$
\frac{1}{\sqrt{p}} e^{ \pm i\left(S+\frac{\pi}{4}\right)} \quad(E>V(x)) \longleftrightarrow \frac{1}{\sqrt{p}}\left(e^{S} \pm \frac{i}{2} e^{-S}\right) \quad(E<V(x))
$$

where

$$
S=\left|\frac{1}{\hbar} \int_{x}^{y_{0}} p d x\right|,
$$

with $y_{0}$ being the classical turning point.

For example, $\phi_{a}$ and $\phi_{b}$ are related as

$$
\begin{aligned}
\phi_{a}(x)= & \frac{1}{\sqrt{p}}\left(c_{1} e^{-\frac{i}{\hbar} \int_{x_{0}}^{x} p d x}+c_{2} e^{\frac{i}{\hbar} \int_{x_{0}}^{x} p d x}\right) \\
\phi_{b}(x)= & \frac{1}{\sqrt{|p|}}\left\{c_{1} e^{-i S_{a}}\left(e^{\frac{1}{\hbar} \int_{x_{1}}^{x} p d x}+\frac{i}{2} e^{-\frac{1}{\hbar} \int_{x_{1}}^{x} p d x}\right)\right. \\
& \left.+c_{2} e^{i S_{a}}\left(e^{\frac{1}{\hbar} \int_{x_{1}}^{x} p d x}-\frac{i}{2} e^{-\frac{1}{\hbar} \int_{x_{1}}^{x} p d x}\right)\right\}
\end{aligned}
$$

In the same way, one obtains the expression for $\phi_{e}(x)$ as

$$
\phi_{e}(x)=\frac{1}{\sqrt{p}}\left\{\left(A c_{1}+B c_{2}\right) e^{-\frac{i}{\hbar} \int_{x_{4}}^{x} p d x}+\left(C c_{1}+D c_{2}\right) e^{\frac{i}{\hbar} \int_{x_{4}}^{x} p d x}\right\}
$$

where $A, B, C$ and $D$ are given as

$$
\begin{aligned}
& A=e^{i S_{a}}\left\{\frac{1}{2}\left(i e^{M_{b}} \sin S_{c}+\frac{1}{4} e^{-M_{b}} \cos S_{c}\right) e^{-M_{d}}+2\left(e^{M_{b}} \cos S_{c}+\frac{i}{4} e^{-M_{b}} \sin S_{c}\right) e^{M_{d}}\right\}, \\
& B=e^{-i S_{a}}\left\{\frac{1}{2}\left(e^{M_{b}} \sin S_{c}+\frac{i}{4} e^{-M_{b}} \cos S_{c}\right) e^{-M_{d}}-2\left(i e^{M_{b}} \cos S_{c}+\frac{1}{4} e^{-M_{b}} \sin S_{c}\right) e^{M_{d}}\right\}, \\
& C=e^{i S_{a}}\left\{\frac{1}{2}\left(e^{M_{b}} \sin S_{c}-\frac{i}{4} e^{-M_{b}} \cos S_{c}\right) e^{-M_{d}}-2\left(-i e^{M_{b}} \cos S_{c}+\frac{1}{4} e^{-M_{b}} \sin S_{c}\right) e^{M_{d}}\right\} \\
& D=e^{-i S_{a}}\left\{\frac{1}{2}\left(-i e^{M_{b}} \sin S_{c}+\frac{1}{4} e^{-M_{b}} \cos S_{c}\right) e^{-M_{d}}+2\left(e^{M_{b}} \cos S_{c}-\frac{i}{4} e^{-M_{b}} \sin S_{c}\right) e^{M_{d}}\right\}
\end{aligned}
$$

On the other hand, with use of the Bloch theorem in Eq.(15) based on the periodicity of the effective potential $V, \phi_{e}$ and $\phi_{a}$ should be related as

$$
\phi_{e}=e^{i k L} \phi_{a},
$$


where $k$ is the wave number. This relation gives a constraint to guarantee the nontrivial coefficients $\left(c_{1}, c_{2}\right)$ of $\phi_{a}$ and $\phi_{e}$ :

$$
\left|\begin{array}{cc}
A-e^{i k L} & B \\
C & D-e^{i k L}
\end{array}\right|=0
$$

which can be rewritten as

$$
e^{i k L}=\cos (k L)+i \sin (k L)=\frac{1}{2}(A+D) \pm i \sqrt{1-\frac{1}{4}(A+D)^{2}} .
$$

Here we have used the fact $A D-B C=1$. At low temperatures the tunneling integrals are sufficiently large, $M_{b} \gg 1$ and $M_{d} \gg 1$. Then we can make the approximation:

$$
\frac{1}{4}(A+D)^{2} \cong 4 e^{2 M_{b}} e^{2 M_{d}} \cos ^{2}\left(S_{a}\right) \cos ^{2}\left(S_{c}\right)
$$

Since (27) should satisfy (26), $\left|\cos S_{a}\right|$ and $\left|\cos S_{c}\right|$ must be sufficiently small and be approximated as

$$
\begin{aligned}
& \cos \left(S_{a, c}\right) \simeq(-1)^{n_{a, c}}\left\{S_{a, c}-\left(n_{a, c}+\frac{1}{2}\right)\right\} \pi \\
& \sin \left(S_{a, c}\right) \simeq(-1)^{n_{a, c}} .
\end{aligned}
$$

Furthermore, using the harmonic approximation in the neighborhood of the potential minima $x_{\text {min }}^{a, c}$, the actions can be evaluated as

$$
S_{a, c}=\frac{\pi}{\hbar \omega_{a, c}}\left(E-V_{0 a, c}\right)
$$

where $\omega_{a, c}=\sqrt{\frac{2 \theta}{\hbar^{2}} V^{\prime \prime}\left(x_{\text {min }}^{a, c}\right)}$ is the curvature of the potential minima, and $V_{0 a, c}$ stands for the local minima of $V(x)$. Then Eq.(26) gives the quantization condition for eigenvalues $E$

$$
\begin{aligned}
& \cos (k L)=\frac{1}{2}(A+D) \\
= & \frac{1}{2}\left\{\left(4 e^{M_{b}+M_{d}}+\frac{1}{4} e^{-M_{b}-M_{d}}\right) \cos \left(S_{a}\right) \cos \left(S_{c}\right)-\left(e^{-M_{b}+M_{d}}+e^{M_{b}-M_{d}}\right) \sin \left(S_{a}\right) \sin \left(S_{c}\right)\right\} \\
\simeq & \frac{1}{2}\left\{\left(4 e^{M_{b}+M_{d}}+\frac{1}{4} e^{-M_{b}-M_{d}}\right)\left(\left(n_{a}+\frac{1}{2}\right) \pi-\frac{\pi}{\hbar \omega_{a}}\left(E-V_{0 a}\right)\right)\left(\left(n_{c}+\frac{1}{2}\right) \pi-\frac{\pi}{\hbar \omega_{c}}\left(E-V_{0 c}\right)\right)\right. \\
& \left.-\left(e^{M_{b}-M_{d}}+e^{-M_{b}+M_{d}}\right)\right\}(-1)^{n_{a}+n_{c}} .
\end{aligned}
$$


Solving the quadratic equation of $E$ in (30), we obtain the lowest few eigenvalues within the $k=0$ manifold,

$$
\begin{aligned}
E_{ \pm} & =E_{0+} \pm \sqrt{E_{0-}^{2}+\epsilon} \\
& \simeq E_{0+} \pm\left(E_{0-}+\frac{\epsilon}{2 E_{0-}}\right)
\end{aligned}
$$

where $E_{0 \pm}$ stand for the harmonic oscillator levels at the wells and $\epsilon$ is a correction due to tunneling, which are defined as

$$
\begin{gathered}
E_{0 \pm}=\frac{1}{2}\left\{V_{0 a} \pm V_{0 c}+\hbar \omega_{a}\left(n_{a}+\frac{1}{2}\right) \pm \hbar \omega_{c}\left(n_{c}+\frac{1}{2}\right)\right\} \\
\epsilon \equiv \epsilon(k=0)=\frac{\hbar^{2} \omega_{a} \omega_{c}}{\pi^{2}} \frac{e^{-2 M_{b}}+e^{-2 M_{d}}+2(-1)^{n_{a}+n_{c}} e^{-M_{b}-M_{d}} \cos k}{4+\frac{1}{4} e^{-2 M_{b}-2 M_{d}}}
\end{gathered}
$$

In general, the eigenvalues form subbands with their width given by the tunneling term $\epsilon$, although we confine ourselves to the $k=0$ state in each of the subbands. At $x_{\min }^{a}=\frac{L}{2 \pi}\left(n \pi-(-1)^{n} \arcsin \frac{W}{U_{0}}\right)$, we find $V_{0 a}=V\left(x_{\min }\right) \simeq$ $-\frac{1}{2}\left(\frac{2 \pi}{L}\right)^{2} \sqrt{1-\left(\frac{W}{U_{0}}\right)} U_{0}$ and

$$
\hbar \omega_{a} \simeq\left(\frac{2 \pi}{L}\right)^{2} \sqrt{1-\left(\frac{W}{U_{0}}\right)^{2}} U_{0} .
$$

Noting further that $V_{0 a}+\frac{1}{2} \hbar \omega_{a} \simeq V_{0 c}+\frac{1}{2} \hbar \omega_{c} \simeq 0$, one can confirm the lowest eigenvalue $E_{-} \simeq 0$, which is responsible to the steady state. Since the tunneling term is small compared to $\hbar \omega_{a}$, one finds the decay rate (:the second-lowest eigenvalue),

$$
\lambda=E_{+} \simeq \hbar \omega_{a}=\left(\frac{2 \pi}{L}\right)^{2} \sqrt{1-\left(\frac{W}{U_{0}}\right)^{2}} U_{0} .
$$

We have confirmed numerically that this expression gives a good approximation for the decay rate in the small tilting case (see Fig.3).

Now we can explain the $W$ (external force) dependence of the decay rate. From (34) one has the monotonic $W$ (external force) dependence of the decay rate. Intuitively, this parameter dependence of the decay rate is explained in terms of tunneling process as follows. The energy level $\hbar \omega_{a}=\sqrt{2 \theta V^{\prime \prime}\left(x_{\text {min }}^{a}\right)}$ is a monotonic function of the curvature of the potential minimum $V^{\prime \prime}\left(x_{\min }^{a}\right)$ which decreases for increasing $W$, because the potential barrier becomes lower and finally the barrier disappears as $W$ increases. The case that each barrier 
separating adjacent local potential minima vanishes is treated in the next section.

\subsection{Large tilting case}

The separation ansatz $P(x, t)=e^{-\frac{U(x)}{2 \theta}} \phi(x) e^{-E t}$ in Eq.(11) seems to contain a divergent term coming from $e^{\frac{2 \pi W x}{2 L \theta}}$ involved in $e^{-\frac{U(x)}{2 \theta}}$. This problem was overcome by choosing the absorbing boundary condition in Eq.(14). Confining ourselves to $k=0$ states as in the previous subsection, we have

$$
\phi(x+L)=e^{-\frac{2 \pi W}{2 \theta}} \phi(x) .
$$

With use of the complex-valued WKB method, Eq. (35) leads to the quantization condition for the complex eigenvalues $E=E_{1}+i E_{2}$ as

$$
e^{ \pm i \int_{0}^{L} d x \sqrt{\theta^{-1}\left(E_{1}-V(x)+i E_{2}\right)}}=e^{-\frac{2 \pi W}{2 \theta}+2 n \pi i}
$$

with $V(x)$ given in Eq.(16).

Remarkably there is no classical turning point on the real axis, and the energy becomes complex, as is confirmed numerically for the strongly tilted case (see Fig.1). Then the decay rate $\lambda$ is given by the real part of the eigenvalue with the second smallest part, which we denote $E_{1}$. The existence of the imaginary part, which we denote $E_{2}$, implies that the asymptotic behavior is oscillating.

Noting again that the second term of (16) is suppressed at low temperatures, the WKB quantization condition (36) is rewritten as

$$
\begin{aligned}
& \frac{\pi W}{\theta L} \int_{0}^{L} \sqrt{1+2 \frac{U_{0}}{W} \sin \frac{2 \pi x}{L}+\left(\frac{U_{0}}{W}\right)^{2} \sin ^{2} \frac{2 \pi x}{L}-\frac{\theta L^{2}}{\pi^{2} W^{2}}\left(E_{1}+i E_{2}\right)} \\
& =-\frac{\pi W}{\theta}+i 2 \pi n .
\end{aligned}
$$

For the large tilting case $W \gg U_{0}$, we can solve this equation perturbatively with the expansion parameter $U_{0} / W$ (see Appendix). Since it is numerically shown that the integer $n$ is \pm 1 for the parameter range $3 \leq U_{0} \leq 20, U_{0} \leq$ $W \leq 20$, we investigate the case that $n= \pm 1$. As a result, we obtain for large $W$

$$
E_{1}=\left(\frac{2 \pi}{L}\right)^{2} \theta
$$




$$
E_{2}= \pm\left(\frac{2 \pi}{L}\right)^{2} W
$$

These results are consistent with those from the numerical diagonalization of FP operator. It is interesting that for large $W$, the decay rate $E_{1}$ saturates and depends only on the temperature, the period and the viscosity. It is also worth noting that $E_{2}$, which represents the oscillation frequency in the asymptotic behavior, is proportional to $W$ and does not depend on $U_{0}$.

\section{Summary}

The low-temperature $\left(\theta=k_{B} T\right)$ decay of the thermal diffusion in the tilted periodic potential (period $L$, amplitude $U_{0}$ and external tilting force $W$ ), which can be described by the Fokker-Planck (FP) equation, is investigated in terms of the (generalized) WKB method. The nonequilibrium steady state demands a subtle approach to the asymptotic decay phenomenon, qualitatively different from the one often used in the context of the decay to the thermal equilibrium. However, we have confined ourselves to the manifold of $k=0$ which includes the steady state. This recourse makes the analysis of the decay phenomenon accessible.

While eigenvalues of FP operator are complex in general, in a small tilting case $\left(W<U_{0}\right)$ the imaginary parts of the eigenvalues are shown to be vanishing. Then the Schrödinger equation associated with FP equation has the WKB solution satisfying PBC. Among the continuum of Bloch states, the zero-wavenumber eigenvalues are essential: the lowest eigevalue is responsible for the steady state and the decay rate is determined by the second-lowest eigenvalue within the zero-wavenumber manifold. The parameter dependence of the decay rate is explained by the tunneling process through a triple-well effective periodic potential. The decay rate $\lambda$ is given by $\left(\frac{2 \pi}{L}\right)^{2} \sqrt{1-\left(\frac{W}{U_{0}}\right)^{2}} U_{0}$. In a large tilting case $\left(W \gg U_{0}\right)$, the imaginary parts of the eigenvalues of FP operator are crucial. We apply the complex-valued WKB method to the Schrödinger equation with the absorbing boundary condition, finding the decay phenomenon characterized by the decay rate $E_{1}=\left(\frac{2 \pi}{L}\right)^{2} \theta$ and the oscillation frequency $E_{2}=\left(\frac{2 \pi}{L}\right)^{2} W$. The intermediate tilting case is also explored, and our theoretical results from the WKB analysis explain $W$ and $U_{0}$ dependence of the numerical decay rate quite well for all parameter ranges in Fig.3. The decay phenomena starting from $k \neq 0$ distributions and the contribution from continuous spectra to nonexponential decays will constitute subjects which we intend to study in future. 


\section{Acknowledgment}

T.M. thanks to Prof. S.Tasaki for valuable comments including the advice about calculation in Eq.(36). This work is partly supported by JSPS Research Fellowship for young scientist and 21st Century COE Program (Holistic research and Education, Culture, Sports, Science and Technology). A.S. and K.N. are grateful to JSPS for the financial support to the Fundamental Research.

\section{A Perturbative analysis of the complex eigenvalue problem for large tilting case}

For the large tilting case $\left(W \gg U_{0}\right)$, the WKB quantization condition under the absorbing boundary condition is given by Eq.(36) or equivalently by

$$
i \int_{0}^{L} d x \sqrt{\theta^{-1}\left(E_{1}-V(x)+i E_{2}\right)}=-\frac{\pi W}{\theta}+2 n \pi i .
$$

We have solved Eq.(A.1) perturbatively with use of the expansion parameter $\epsilon=\frac{U_{0}}{W}$ as follows.

We restrict ourselves to the case $n= \pm 1$ which is observed numerically in the parameter range $3 \leq U_{0} \leq 20, U_{0} \leq W \leq 20$ and use a branch of square-root with positive imaginary part in the left hand side of (A.1) (another branch does not satisfy (A.1)). At first, we note that (A.1) is equivalent to

$$
\begin{aligned}
& -\int_{0}^{L} d x\left(\sqrt{\frac{1}{2}\left(A+\sqrt{A^{2}+B^{2}}\right)}+i \sqrt{\frac{1}{2}\left(-A+\sqrt{A^{2}+B^{2}}\right)}\right) \\
= & -L+i \frac{2 \theta L}{W} \\
A= & \left(1+\epsilon \sin \frac{2 \pi x}{L}\right)^{2}-\frac{\theta L^{2}}{\pi^{2} W^{2}} E_{1} \\
B= & \frac{\theta L^{2}}{\pi^{2} W^{2}} E_{2} .
\end{aligned}
$$

We expand eigenvalue $E_{1}+i E_{2}$ up to the second order of $\epsilon$ as

$$
\frac{\theta L^{2}}{\pi^{2} W^{2}} E_{1}=A_{1} \epsilon+A_{2} \epsilon^{2}+O\left(\epsilon^{3}\right), \quad \frac{\theta L^{2}}{\pi^{2} W^{2}} E_{2}=B_{1} \epsilon+B_{2} \epsilon^{2}+O\left(\epsilon^{3}\right) .
$$


Then the real part of the equation (A.2) is expanded in $\epsilon$ as

$$
\begin{aligned}
& \int_{0}^{L} d x\left\{1+\frac{1}{2}\left(-A_{1}+2 \sin \frac{2 \pi x}{L}\right) \epsilon\right. \\
& \left.+\left(\frac{-1}{8}\left(-A_{1}+2 \sin \frac{2 \pi x}{L}\right)^{2}-\frac{1}{2} A_{2}+\frac{1}{2} \sin ^{2} \frac{2 \pi x}{L}+\frac{1}{8} B_{1}^{2}\right) \epsilon^{2}+O\left(\epsilon^{3}\right)\right\}=L .
\end{aligned}
$$

Noting that coefficients of each power of $\epsilon$ should vanish and using $\int_{0}^{L} d x \sin \frac{2 \pi x}{L}=$ $0, \int_{0}^{L} d x \sin ^{2} \frac{2 \pi x}{L}=\frac{1}{2}$, we obtain

$$
A_{1}=0, \quad A_{2}=\frac{1}{4} B_{1}^{2} .
$$

In the same way, the imaginary part of (A.2) is (provided $A_{1}=0$ )

$$
\int_{0}^{L} d x\left\{\frac{B_{1}}{2} \epsilon+\frac{1}{2}\left(B_{2}-B_{1} \sin \frac{2 \pi x}{L}\right) \epsilon^{2}+O\left(\epsilon^{3}\right)\right\}=\frac{2 \theta L}{W} .
$$

Thus one has

$$
A_{2}=\frac{4 \theta^{2}}{U_{0}^{2}}, \quad B_{1}= \pm \frac{4 \theta}{U_{0}}
$$

From these expressions the eigenvalue is given by Eq.(38). As shown in Fig.3 this estimation of $E_{1}$ is very accurate for $W \gg U_{0}$.

\section{References}

[1] J. W. M. Frenken and J. F. Van der Veen, Phys. Rev. Lett. 54, (1985) 134; B. Pluis et al., ibid. 59 (1987) 2678 ; T. R. Linderoth, S.Horch, E. Laegsgaard, I. Stensgaard, and F. Besenbacher, ibid.78 (1997) 4978; P. Talkner, E. Hershkovitz, E. Pollak, and P. Hänggi, Surf. Sci. 437 (1999) 198

[2] A. Barone and G. Paterno, Physics and Applications of the Josephson Effect (Wiley, New York, 1982)

[3] B. Shapiro, M. Gitterman, I. Dayan, and G. H. Weiss, Phys. Rev B 46 (1992) 8416

[4] A. Burshtein and Y. Georgievskii, J. Chem. Phys. 100 (1994) 7319 
[5] P. Fulde, L. Pietronero, W. R. Schneider, and S. Strässler, Phys. Rev. Lett. 35 (1975) 1776; W. Dietrich, P. Fulde, and I. Peschel, Adv. Phys. 29 (1980) 527

[6] G. Gruner, A. Zawadowski, and P. M. Chaikin, Phys. Rev. Lett. 46 (1981) 511

[7] A. Ajdari and J. Prost, Proc. Natl. Acad. Sci. U. S. A. 88 (1991) 4468; G. I. Nixon and G. W. Slater, Phys. Rev. E 53 (1996) 4969

[8] F. C. Hoppenstead and E. M. Izhikevich, Weakly Connected Neural Networks (Springer, New York, 1997); K. Wiesenfeld, D. Pierson, E. Pantazelou, C. Dames, and F. Moss, Phys. Rev. Lett. 72 (1994) 2125 ; C. Kurrer and K. Schulten, Phys. Rev. E 51 (1995) 6213

[9] P. Reimann, Phys. Rep. 361 (2002) 57

[10] P. Hänggi, P. Talkner, and M. Borkovec, Rev. Mod. Phys. 62 (1990) 251

[11] C. R. Doering, W. Horshtemke, and J. Riordan, Phys. Rev. Lett. 72 (1994) 2984

[12] P. Reimann, C. V. Broeck, H. Linke, P. Hänggi, J. M. Rubi, and A. PérezMadrid, Phys. Rev. Lett. 87 (2001) 010602; P.Reimann, C.V.Broeck, H.Linke, P. Hängi, J.M.Rubi, and A.Pérez-Madrid, Phys.Rev.E 65 (2002) 031104

[13] H. Risken, The Fokker Planck Equation ( Springer, Berlin, 1989)

[14] J. L. Lebowitz and P. G. Bergmann, Ann. Physik 1 (1957) 1

[15] N. G. van Kampen, J. Stat. Phys. 17 (1977) 71; Supplement of Prog. Theor. Phys. 64 (1978) 389; Stochastic processes in physics and chemistry, 2nd ed. (North-Holland, Amsterdam, 1981)

[16] B.Caroli,C.Caroli,and B.Roulet, J. Stat. Phys. 21 (1979) 415; B. Caroli, C. Caroli, and B. Roulet, J. Stat. Phys. 26 (1981) 83; H. Tomita, A. Ito,and H. Kidachi, Prog. Theor. Phys. 56 (1976) 786; K. Nakamura and T. Sasada, Phys. Lett. A. 74 (1979) 379

[17] P. Noziére and G. Iche, J. Physique 40 (1979) 225; W. Dieterich et al., Z. Physik B 27 (1977) 177

[18] D ter Haar, Selected Problems in Quantum Mechanics (Academic, New York, 1964); S. Ohta and K. Nakamura, J. Phys. C 14 (1981) L427 